\title{
Epidemiología del uso/abuso de cannabis
}

\author{
Bobes, J.*; Bascarán, M.T**.; González, M.P.**; SÁlz, P.A.**** \\ * Catedrático de Psiquiatría. Area de Psiquiatría. Universidad de Oviedo. \\ * Colaboradora de Honor. Area de Psiquiatría. Universidad de Oviedo. \\ ** Prof. Titular de Psicología Médica. Area de Psiquiatría. Universidad de Oviedo. \\ *** Prof. Asociado de Psicología Médica. Area de Psiquiatría. Universidad de Oviedo. \\ Enviar correspondencia a: J. Bobes García. Universidad de Oviedo. Fac. Medicina - Area de Psiquiatría. Julián Clavería, 6. 33006 Oviedo.
} e-mail: bobes@correo.uniovi.es

\section{Resumen}

En este capítulo se realiza una revisión y análisis comparativo de los datos obtenidos en los estudios epidemiológicos realizados por los organismos nacionales e internacionales de más prestigio (Plan Nacional de Drogas, Observatorio Europeo de las Drogas y las Toxicomanías, y el Grupo de Trabajo Internacional en la Epidemiología de las Drogas de Abuso del Instituto Nacional de la Salud Estadounidense).

Por otra parte se aportan nuestros datos de consumo en población recluta, en estudiantes de secundaria y en estudiantes universitarios de Ciencias de la Salud de Oviedo.

Asimismo, se facilitan datos que señalan la magnitud e importancia de los derivados cannábicos como desencadenantes de trastornos psicóticos y agravantes de los trastornos esquizofrénicos.

Finalmente, se recogen los resultados más sobresalientes del informe sobre la percepción de riesgos relacionados con las drogas y su problemática, realizado por la Fundación de Ayuda contra la Drogodependencia.

Palabras clave: epidemiología, cannabis, comorbilidad, percepción social.

\section{Summary}

A review and comparative analysis was made of data obtained from the most important epidemiological studies carried out by the most prestigious national and international organizations in the last decade (Plan Nacional sobre Drogas, European Monitoring Centre for Drugs and Drug Addiction, and the International Epidemiology Work Group on Drug Abuse $\mathrm{NIH)}$.

We also include our data obtained a from military recruits, secondary school students, and university undergraduate Helath Science students.

Furthermore, we include information of the impact of cannabis in the psychogenesis and comorbidity of schizophrenic patients.

Finally, we summarize the results of a Spanish general population study obtained by the Fundación de Ayuda contra la Drogadicción about risk perception and the consequences of use and abuse of cannabis.

Key words: epidemiology, cannabis, comorbidity, social perceptions.

\section{TENDENCIAS EN EL USO/ABUSO DE CANNABIS EN LA UNIÓN EUROPEA}

P ara describir y analizar la tendencia actual de uso/abuso del cannabis en el contexto europeo creemos que lo más adecuado es seguir la información del Centro Europeo para la Monitorización de las Drogas y la Drogadicción (EMCDDA) (1). El EMCDDA es una agencia de la Unión Europea que tiene como objetivo proporcionar una visión objetiva, fiable, y comparable sobre las dro- 
gas, la adicción a las mismas, y las consecuencias de su abuso a nivel de la Unión Europea.

La droga ilícita más consumida en la Unión Europea es el cannabis, estimándose en aproximadamente más de 40 millones el número de personas que lo han usado, y en al menos 12 millones el número de personas que lo han usado en el último año. Es decir, aproximadamente el $16 \%$ de la población de la Unión Europea de edades comprendidas entre los 15 y los 64 años habrían consumido cannabis alguna vez en su vida, y cerca del $5 \%$ lo habrían consumido en el último año.

Las tasas de prevalencia de consumo varían de forma importante en función de la edad, aumentando considerablemente entre los más jóvenes. Así, los datos del European School Survey on Alcohol and Others Drugs (2) realizado en el año 1995 en 25 países de Europa, indican que aproximadamente el $20 \%$ de los adolescentes entre 15 y 16 años han consumido cannabis alguna vez en su vida, y se estima que cuando alcancen los 25 años el porcentaje alcanzará al menos al 30\% de ellos. En los países de Europa central y oriental, con cifras algo más bajas que las de la Europa occidental (entre el 7 y el 13\%), se está produciendo un incremento constante en las tasas.

Al igual que lo sucedido con el alcohol, estamos asistiendo a un fenómeno de homo- geneización de las tasas de consumo entre los distntos países, de tal modo que en los países con tasas más elevadas el consumo se ha estabilizado o incluso disminuido, mientras que éste ha aumentado en los países que tradicionalmente poseían tasas más bajas. Sin embargo, el análisis global de la situación en Europa indica que en estos últimos 10 años estamos asistiendo a un incremento neto en el uso de cannabis, y que este incremento es el mayor del planeta (3).

A pesar del fenómeno de la homogeneización de las tasas en Europa, todavía existen diferencias sustanciales en cuanto a la magnitud del consumo entre los distintos países. Así por ejemplo, para los sujetos de edades comprendidas entre los 15 y 35 años (adolescentes y adultos jóvenes) la tasa de prevalencia vida oscila entre el 16 y el $43 \%$, y la tasa de prevalencia en el último año entre el 2 y el $21 \%$.

Cuando se comparan los datos de la Encuesta de Población sobre el consumo de cannabis por los adultos de los Estados miembros de la Unión Europea (4) nos encontramos que España está a la cabeza del "ranking", únicamente superada por Dinamarca y el Reino Unido. El $22 \%$ de todos los adultos españoles y el $32 \%$ del subgrupo de adultos jóvenes han consumido cannabis alguna vez en su vida (Figura 1).

\section{Figura 1. Prevalencia vida del consumo de cannabis en los Estados miembros de la Unión Europea.}

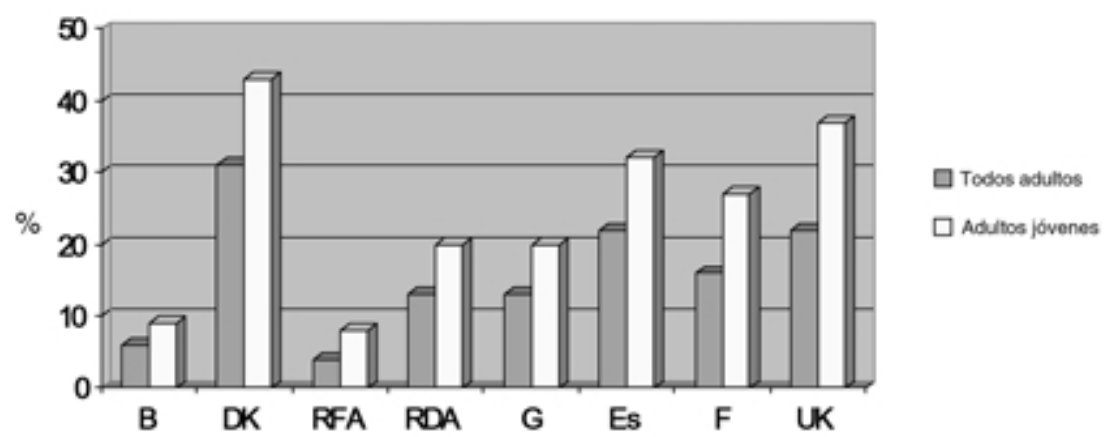

B. Bélgica; DK. Dinamarca; RFA. República Federal de Alemania; RDA. República Democrática de Alemania; G. Grecia; Es. España; F. Francia; UK. Reino Unido. 
Además de estos datos sobre la extensión del uso, el EMCDDA ha detectado un incremento leve en la demanda de tratamiento debido al uso de cannabis en algunos países. En la actualidad, aproximadamente el 10\% de las demandas de tratamiento tienen como droga principal al cannabis, siendo este porcentaje mayor en los nuevos pacientes que demandan tratamiento por primera vez. Se desconocen las razones exactas de este incremento, argumentando el EMCDDA que podría deberse en parte al aumento de uso, pero que también estarían implicados una serie de factores ajenos como son cambios en el sistema judicial (sentencias de tratamiento en vez de privación de libertad), etc.

Cuando analiza los decomisos de cannabis, el EMCDDA observa que si bien el número/ año está aumentando progresivamente la cantidad decomisada/año permanece estable. La disponibilidad es elevada en la mayor parte de los países de la Unión Europea, y el precio permanece estable.

\section{TENDENCIAS EN EL USO/ABUSO DE CANNABIS EN ESPAÑA.}

Al igual que en el apartado anterior haremos especial referencia a los datos obtenidos por el Plan Nacional de Drogas a través de sus encuestas: La Encuesta sobre Drogas a la Población Escolar (se realiza los años pares) y La Encuesta Domiciliaria sobre Consumo de Drogas (se realiza los años impares). Dadas las diferencias metodológicas entre ambas (algunas tan obvias como la edad) presentaremos por separado los resultados de ambas.

\subsection{Encuesta sobre Drogas a la Población Escolar (PND, 1994(5), 1996(6), 1998(7).}

Esta encuesta se viene realizando de forma periódica cada 2 años y en ella participan los estudiantes de los centros públicos y privados de enseñanza secundaria y formación profesional, con edades comprendidas entre los 14 y los 18 años.
Con respecto al porcentaje de consumidores se ha venido experimentando un incremento tanto del consumo experimental como del consumo más regular, aunque es necesario señalar que el incremento de las tasas ha sido mucho mayor entre el año 94 y el 96 que entre el 96 y el 98. Así, la prevalencia vida pasó del $20.8 \%$ en el año 94, al 26\% en el año 96 , y al $28.5 \%$ en el año 98 . Comparadas estas cifras con las de Europa occidental se demuestra claramente como nuestro país se sitúa en los primeros puestos, por encima de la media señalada en el epígrafe anterior. El porcentaje de consumidores en los últimos 12 meses fue del $18.1 \%$ en el año 94 , del $23.2 \%$ en el año 96 , y del $25.1 \%$ en el año 98. La prevalencia del consumo en los últimos 30 días fue del $15.7 \%$ en el año 96 y del $17.2 \%$ en el año 98.

A medida que aumenta la edad se observa un incremento en la proporción de adolescentes que consumen cannabis (Figura 2). En esta figura también se puede observar como el incremento en el consumo en los últimos años se produce con más intensidad entre los más jóvenes. Por sexos, los hombres presentan tasas superiores a las mujeres tanto en la prevalencia vida como en los últimos 12 meses en las tres encuestas, sin que parezca que se estén produciendo cambios importantes en este sentido.

En cuanto a la edad media de inicio al consumo del cannabis se observa una tendencia al descenso de la misma, pasando de 15.1 años en el año 94, a 15 años en el 96, y a 14.8 años en el 98.

\subsection{Encuesta Domiciliaria sobre Consumo de Drogas (PND, 1995(8), 1997(9).}

Esta encuesta al igual que la anterior se viene realizando de forma periódica cada dos años entre los residentes en el territorio nacional incluyendo Ceuta y Melilla. Existen algunas diferencias metodológicas importantes entre ambas. En primer lugar, el grupo de edad estudiado varía, no teniendo en el año 95 límite superior (se incluyeron personas 
Figura 2. Tendencia del consumo en los últimos 12 meses en función de la edad (Datos de la Encuesta sobre Drogas a la Población Escolar, años 1994, 1996, y 1998).

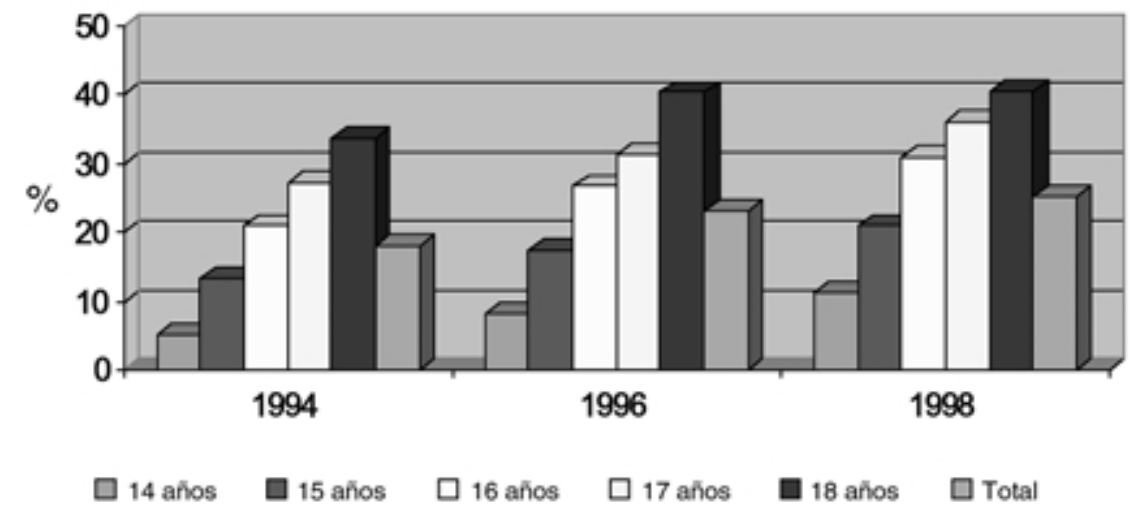

mayores de 14 años) y sí en el año 97 (se incluyeron personas de edades comprendidas entre los 15 y 65 años). En segundo lugar, el método de recoger la información sobre el consumo también cambió, utilizándose el método de entrevista en el 95 y de autoinforme en el 97. Teniendo en cuenta estas diferencias que dificultan en cierta medida las comparaciones directas pasamos a resumir los principales resultados hallados.

Entre estos dos años se observa un aumento en las tasas de consumos experimentales u ocasionales y un descenso en el consumo habitual (diario) (figura 3). Como comentábamos anteriormente, las diferencias metodológicas entre las dos encuestas hacen que esta comparación simple sea tenida en cuenta con ciertas limitaciones, ya que el hecho de incluir en la encuesta del 95 a personas de más de 65 años, entre las cuales las tasas de consumo son prácticamente inexistentes, haría descender las tasas totales de prevalencia en este año respecto al 97. Sin embargo, esta influencia de la edad creemos que no explicaría todo el aumento, ya que en el caso de los consumos habituales la

Figura 3. Tendencias de los consumos de cannabis en población española. (Datos de la Domiciliaria sobre Consumo de Drogas, años 1995 y 1997).

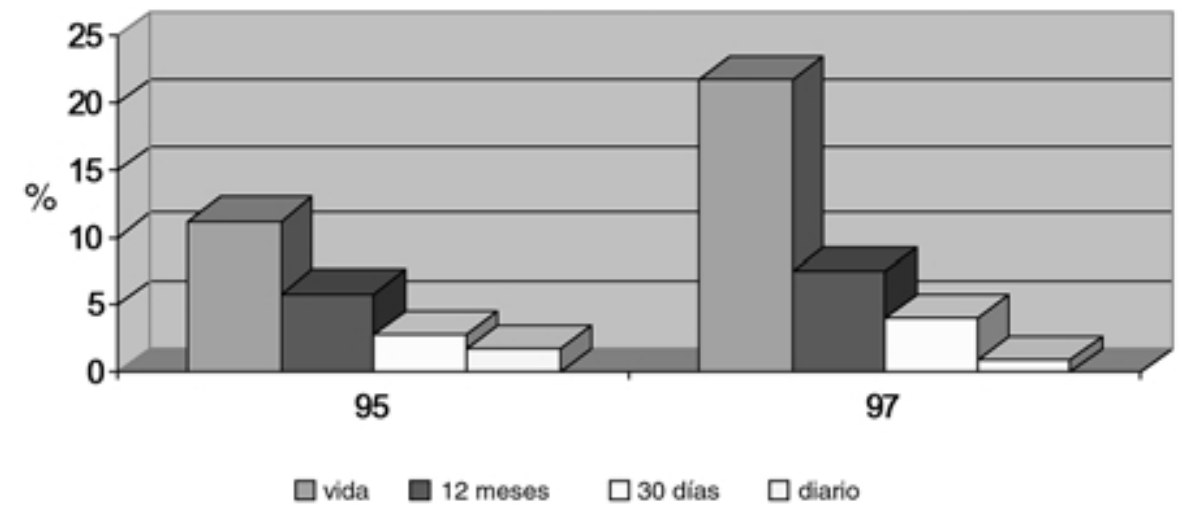


variación entre los dos años se ha producido en sentido inverso al que cabría esperar si las diferencias en las edades estudiadas fueran las responsables al ciento por ciento de los cambios experimentados entre los dos años.

En los datos del año 97, se puede apreciar como las mayores tasas de prevalencia vida se dan entre los adultos jóvenes; en concreto el grupo de edad de 19 a 28 años es el que presenta prevalencias más altas (36.1\%), seguido del grupo de 29 a 38 años (31.3\%).

Con respecto a la edad de inicio también ha habido ligeros cambios, $y$, sorprendentemente, éstos son contrarios a la tendencia general observada en la Encuesta a estudiantes y en los estudios internacionales: se ha producido un ligero aumento en la edad media de inicio al consumo pasando de 18.21 años en la encuesta del año 95 a 18.9 años en la encuesta del 97 se observa como entre los grupos de edad más jóvenes la edad de inicio es menor; así entre los adolescentes de 15 a 18 años la edad de inicio es de 15.6 años, entre los jóvenes de 19 a 28 años la edad de inicio es de 17.4 años, entre los jóvenes de 29 a 38 años des de 18.7, entre los adultos de 39 a 48 años ese de 22 años, y finalmente entre los mayores de 48 años la edad media de inicio es de 28.1 años. Es decir, estos datos confirman la tendencia de que estamos asistiendo a un descenso progresivo de la edad en la que los adolescentes inician el consumo de drogas.

\subsection{Datos de consumo en nuestra comuni- dad (Principado de Asturias).}

Desde el año 1995 hemos venido realizando repetidamente un estudio sobre la preva- lencia del consumo de tabaco, alcohol, y otras drogas entre los reclutas del Regimiento Príncipe de Asturias de Noreña. Cada reemplazo rellenaba en los primeros días de su incorporación la Encuesta sobre Drogas de la OMS. Un total de 3.634 reclutas cumplimentaron la encuesta. Con esta misma metodología hemos realizado un estudio sobre los datos de consumo en los estudiantes de secundaria de una zona industrial de Asturias (Langreo) y hemos iniciado el curso pasado encuestas sobre el consumo entre la población estudiantil de secundaria de los Institutos de Oviedo, y con anterioridad a los estudiantes universitarios de Ciencias de la Salud.

\subsection{Población recluta.}

Su edad media era de 20.28 años y el $100 \%$ eran varones. En conjunto, la prevalencia vida de consumo de cannabis era del $40 \%$, la prevalencia en los últimos 12 meses del $25.9 \%$, y en los últimos 30 días del $17.3 \%$. Las tasas de prevalencia por año se expresan en la tabla 1. De los datos de esta tabla se deduce como los consumos se han venido mateniendo prácticamente estabilizados a lo largo de los 5 años de estudio.

La comparación de estos datos con los del ámbito nacional resulta problemática por las diferencias de edad y de sexo (en los reclutas tan sólo hombres). Si los comparamos con los de las encuestas a población estudiantil, y dentro de éstas con el grupo de edad más parecido a la media de nuestros reclutas, es decir, los estudiantes de 18 años, obtenemos que en los mismos años las tasas de prevalencia en los últimos 12 meses son menores entre los

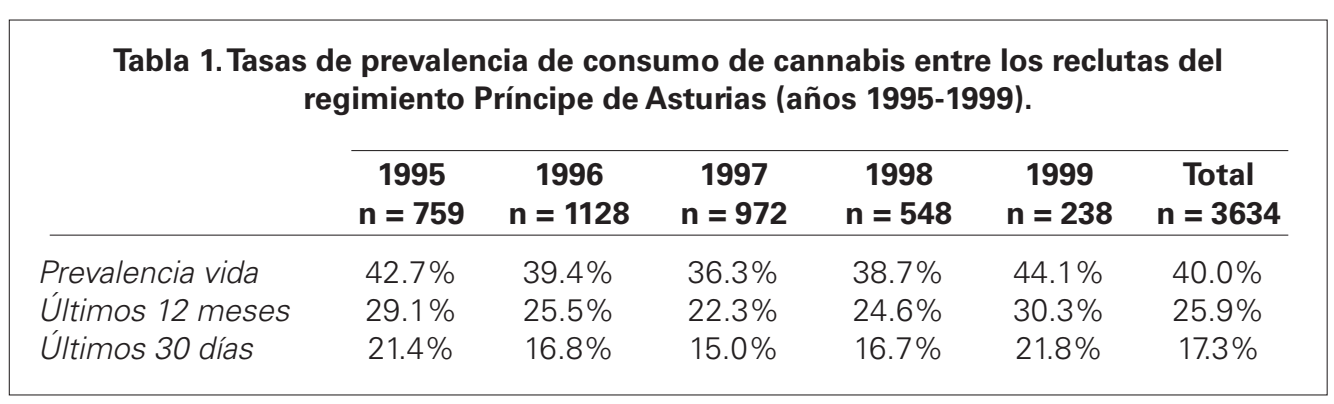


reclutas que entre los estudiantes (año 1996: reclutas $25.5 \%$, estudiantes $40.3 \%$; año 1998 : reclutas $24.6 \%$, estudiantes: $40.6 \%$ ). Comparados con los datos de la encuesta sobre población general del año 97 para el grupo de edad 19-28 años, se observan como aunque las tasas de consumo experimental son iguales, los consumos habituales son más frecuentes entre la población recluta que entre la población general (prevalencia vida: reclutas: $36.3 \%$, población general 19-28 años: $36.1 \%$; prevalencia últimos 30 días: reclutas: 15\%, población general 19-28 años: 9.4\%).

La edad media de inicio al consumo se sitúa entre la de los escolares españoles y la de la población general, siendo para el conjunto de los reclutas de 16.04 años. Por años, prácticamente no ha variado, si acaso se experimentó un ligero aumento entre el año 95 y 96 (edad inicio en el año 95: 15.6, año
96: 16.1; año 97: 16; año: 98: 16.2, y año 99: 16 años).

Dada la abundancia de literatura que señala al cannabis como droga de puerta de entrada a otras drogas, nos pareció interesante calcular el riesgo (razón de ventaja u odds ratio) que poseían nuestros consumidores de cannabis para consumir otras drogas ilegales. Tal como puede observarse en la tabla 2, los reclutas que han consumido cannabis alguna vez en su vida tienen un elevado riesgo de haber consumido otras drogas ilegales. La droga con más probabilidades de haber consumido son los alucinógenos (38 veces más probabilidades) seguidos de la cocaína (36 veces más probabilidades), el éxtasis (33 veces más probabilidades), y las anfetaminas (19 veces más probabilidades). El riesgo para estos reclutas de haber consumido cualquier otra droga ilegal es 12 veces mayor.

Tabla 2. Estudiantes de secundaria: riesgo (odss ratio) de los consumidores de cannabis alguna vez en la vida de consumir cada una de las otras drogas alguna vez en la vida.

\begin{tabular}{lcccr}
\cline { 2 - 5 } & \multicolumn{2}{c}{ Odss ratio } & \multicolumn{2}{c}{ I.C. 95\% } \\
& Estudiantes & Militares & Estudiantes & \multicolumn{1}{c}{ Militares } \\
\hline Cocaína & 30.729 & 36.736 & $16.593-56.909$ & $24.184-55.800$ \\
Anfetaminas & 19.309 & 19.677 & $12.648-29.477$ & $14.342-26.998$ \\
Alucinógenos & 24.737 & 38.361 & $16.611-36.840$ & $25.836-56.957$ \\
Inhalantes & 7.167 & 14.453 & $5.213-9.854$ & $9.147-22.838$ \\
Tranquilizantes & 4.240 & 3.914 & $3.293-5.460$ & $3.082-4.970$ \\
Sedantes & 7.792 & 8.361 & $4.011-15.136$ & $4.707-14.855$ \\
Heroína & 13.068 & 15.580 & $5.528-30.892$ & $8.099-29.971$ \\
Éxtasis & 9.321 & 33.362 & $5.643-15.396$ & $21.565-51.613$ \\
Cualquier otra droga ilegal & 8.522 & 12.677 & $6.918-10.497$ & $10.495-15.313$
\end{tabular}

\subsubsection{Estudiantes de secundaria de Lan- greo (10).}

Cumplimentaron la encuesta 816 estudiantes de secundaria de los colegios públicos y privados de Langreo. La edad media era de 15.9 (1.37) años y el $50.9 \%$ eran hombres. La prevalencia vida de consumo fue del $29.3 \%$ en los últimos 12 meses, del $24.1 \%$ y en el último mes del 9.3\%. La edad media de inicio al consumo de cannabis fue de 15 (1.6) años. No se hallaron diferencias estadísticamente significativas en cuanto a las tasas de consumo ni en función del sexo ni del tipo de centro, privado o público.

\subsubsection{Estudiantes de secundaria de Oviedo.}

Un total de 2.862 estudiantes cumplimentaron la encuesta. Su edad media fue de 15.8 (1.4) años, y el $50.6 \%$ eran varones. El $28.4 \%$ cursaba $1^{\circ}$ de bachiller, el $6.1 \% 2^{\circ}$ de la ESO, 
el $33.8 \% 3^{\circ}$ de la ESO, el $28.9 \% 4^{\circ}$ de la ESO, y el $2.8 \%$ módulos de formación profesional.

La prevalencia vida fue del $35.6 \%$, la prevalencia en los 12 últimos meses del 28\%, y en los últimos 30 días del 21.2\%. La edad media de inicio al consumo era de 14.6 años. Unicamente se encontraron diferencias significativas en función del sexo en la prevalencia en los últimos 30 días, de tal forma que en las mujeres ésta era significativamente más baja que en los hombres (p.01) (Figura 4). La edad media de inicio al consumo fue de 14.6 años, sin diferencias en función del sexo (14.5 para los varones y 14.7 para las mujeres).

Con respecto a la escalada de drogas, tal como puede observarse en la tabla 2, al igual que en el caso de los reclutas, entre los estudiantes de secundaria el hecho de haber consumido cannabis alguna vez en su vida supone un riesgo de magnitud considerable para el consumo alguna vez en la vida de todas las demás drogas evaluadas. En concreto, un estudiante que haya consumido alguna vez en la vida cannabis tiene 30 veces más probabilidades de haber consumido cocaína alguna vez en la vida, casi 25 veces más probabilidades de haber consumido alucinógenos alguna vez en la vida, 19 veces más probabilidades de haber consumido heroína alguna vez en la vida, etc. En resumen, es 8 veces más probable que los estudiantes que consumieron can- nabis alguna vez en su vida hayan consumido cualquier otra droga ilegal. Salvo, en el caso del éxtasis los riesgos son muy similares entre los reclutas y los estudiantes, y pensamos que las diferencias son debidas, al menos parcialmente, a las diferencias de edad. Los riesgos que calculamos son para haber consumido alguna vez en la vida, y los reclutas al tener una edad media mayor que los estudiantes han tenido más tiempo de acercarse a las distintas drogas, por lo que parece lógico que los riesgos sean algo mayores.

\subsubsection{Estudiantes de Ciencias de la Salud del distrito universitario de Oviedo.}

Durante el curso académico 1996-97 un total de 410 alumnos de la Facultad de Medicina y de las Escuelas Universitarias de Enfermería y Fisioterapia completaron la encuesta sobre consumo de tabaco, alcohol, y otras drogas. La edad media era de 21.5 años y el $80.5 \%$ eran mujeres.

La prevalencia vida fue de $29.8 \%$, en los últimos 12 meses del 19\%, y en el último mes del $8.5 \%$. Al igual que en el caso de los estudiantes de secundaria únicamente se encontraron diferencias estadísticamente significativas en función del sexo en el consumo en el último mes, presentando las mujeres

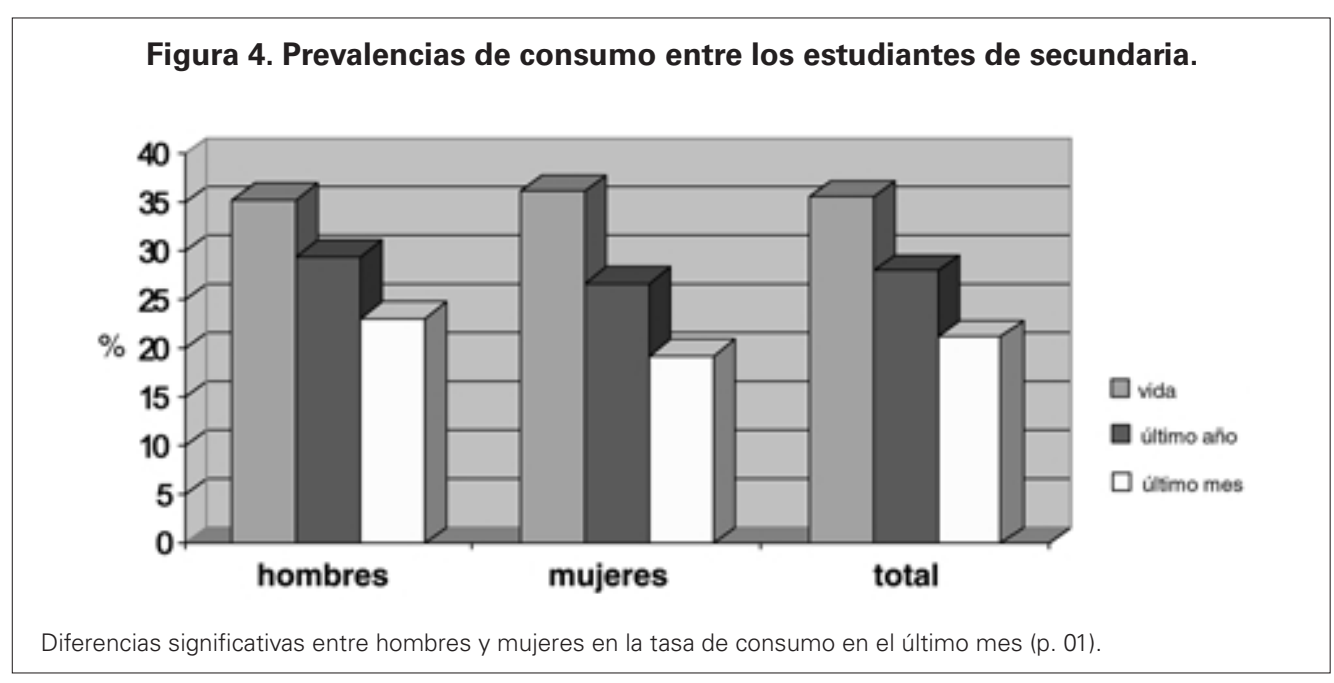


una tasa significativamente inferior a la de los hombres (p. 01) (Figura 5).

La edad media de inicio para el conjunto de los estudiantes era de 17.5 años, sin diferencias en función del sexo (17.5 para los hombres y 17.4 para las mujeres). Tal como cabía esperar, al ser mayor la edad media de este grupo, la edad media de inicio es superior a la de los estudiantes de secundaria.

\subsection{Datos de consumo en pacientes con trastornos esquizofrénicos.}

La asociación entre los trastornos esquizofrénicos y el consumo de cannabis es un tema ampliamente debatido en la literatura científica. Por ello, nos ha parecido conveniente traer brevemente a colación los datos de consumo de cannabis de dos estudios multicéntricos realizados en nuestro país en los que se estudiaron 377 y 353 pacientes esquizofrénicos (criterios CIE-10), obteniéndose una frecuencia de consumo de cannabis entre el 7 y el $13 \%$.

\section{Percepción subjetiva de riesgos.}

En el año 1998 la Fundación de Ayuda contra la Drogadicción (FAD) realizó una encues- ta para determinar la percepción social de los problemas de drogas en España (11). Los datos más destacados con respecto a la percepción social de los problemas asociados al consumo de cannabis se refieren a continuación.

La encuesta se realizó a una muestra representativa de la población de 15 a 65 años residente en el territorio español. El tamaño muestral fue de 1700 sujetos.

Con respecto al cannabis el $75 \%$ de los entrevistados conocían su nombre y lo referían cuando se les preguntaba que citasen los nombres de drogas que les sonasen o conociesen. Fue la droga citada por un mayor número de entrevistados tras la cocaína (y crack) que fue citada por el $77.2 \%$ de la muestra.

El $64 \%$ de los entrevistados cree que el cannabis es la droga que más se consume en nuestro país, le sigue en importancia el alcohol que es contestado por el $61.2 \%$ de los entrevistados. El 27.3\% de los encuestados refirió haber consumido alguna vez cannabis y el $83.2 \%$ alcohol. Es decir, parece que existe una sobreestimación del consumo del cannabis por parte de la población.

Con respecto a la percepción y valoración de la peligrosidad de las drogas, la mayoría de los encuestados opina que las drogas son

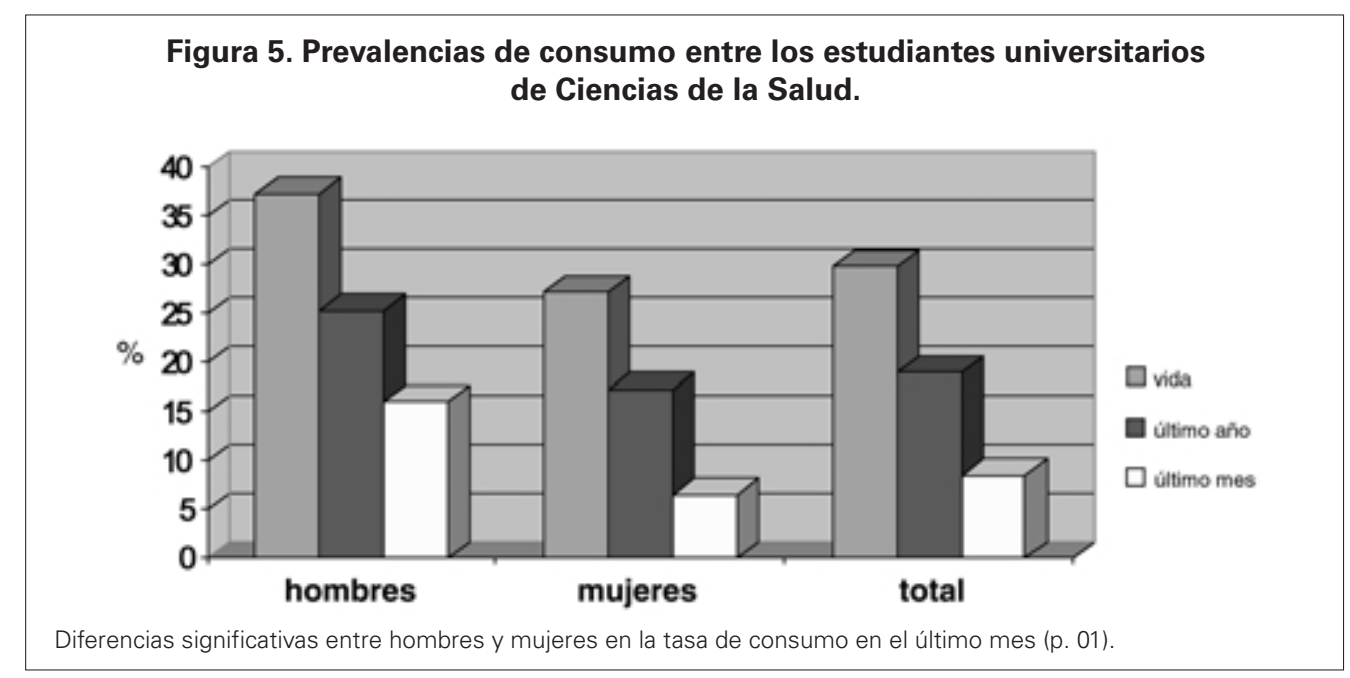


"sustancias muy peligrosas que no deben ni probarse" (74.9\%), y tan sólo el 9.9\% opina que son "algo que es preciso conocer y probar, al menos una vez". Tras el tabaco (puntuación de 2.22) y el alcohol (puntuación de 2.37), el cannabis fue considerada la droga menos peligrosa (puntuación de 2.48 es una escala de $1=$ poco 0 nada peligrosa a $3=$ bastante o muy peligrosa). El $11.6 \%$ de los entrevistados opinaron que era poco o nada peligrosa, el $27.3 \%$ que era regular de peligrosa, y el $58.4 \%$ que era bastante o muy peligrosa. Teniendo en cuenta los resultados de un análisis factorial, los autores concluyen que la posición respecto a la peligrosidad de cannabis es ambivalente ya que tendría aproximadamente el mismo peso en los dos factores obtenidos; en el factor 1 de drogas más peligrosas (cocaína y crack, heroína y otros opiáceos, anfetaminas-alucinógenos y drogas de síntesis, y tranquilizantes e hipnóticos: su peso es de .408, y en el factor 2 de drogas menos peligrosas (tabaco y alcohol) su peso es de 445 .

La actitud de los encuestados frente al cannabis es bastante permisiva, ya que el $39 \%$ opina que se debería permitir su consumo, y el $37.6 \%$ que se debería permitir su venta.

\section{CONCLUSIONES}

- España se sitúa en la actualidad entre los países europeos con mayores tasas de consumo (uso-abuso) de cannabis.

- Las tasas de consumo de cannabis han ido incrementándose progresivamente en las últimas décadas, si bien desde el año 1995 puede considerarse que permanecen prácticamente estables en nuestro país.

- Se observa una tendencia a que el inicio al consumo se realice a edades más tempranas.

- Se confirma el calificativo de droga de "acceso" o "puerta de entrada" al consumo de otras drogas. El riesgo de consumir otras drogas ilegales si se consume cannabis es muy elevado.

- La sociedad española "conoce" esta droga, y la percibe como poco peligrosa, y por tanto, con bajos riesgos.

- Un importante porcentaje de la sociedad española considera que debería permitirse tanto el consumo como la venta de esta sustancia.

- Finalmente, en estos momentos en que las instituciones de más prestigio en medicina consideran muy necesaria la realización de estudios que permitan conocer en profundidad el valor terapéutico para uso médico (12), creemos que estamos ante un grupo de drogas capaces de generar dependencia, alteraciones afectivas, cognitivas, y sensoperceptivas, y agravación del estado mental de personas vulnerables y enfermos psiquiátricos, por lo que no consideramos pertinente "banalizar" su uso-abuso.

\section{BIBLIOGRAFÍA}

(1) EMCDDA: Estimating the prevalence of problem drug use in Europe. EMCDDA Scientific Monograph Series, № 1.

(2) Björn Hibell y cols.: The 1995 ESPAD report: alcohol and other drug use among students in 26 European countries. Estocolmo: Swedish Council for Information on Alcohol and Other Drugs (CAN) y el Pompidou Group. 1997.

(3) National Institute on Drug Abuse: Epidemiological trends in drug abuse. NIH Publication No. 00-4530. Maryland: NIH. 1999.

(4) OEDT: Informe anual sobre el problema de la drogodependencia en la Unión Europea. Bélgica: OEDT. 1999.

(5) Plan Nacional sobre Drogas: Encuesta sobre drogas a la población escolar, 1994. Madrid: Delegación del Gobierno para el Plan Nacional sobre Drogas. 1995.

(6) Plan Nacional sobre Drogas: Encuesta sobre drogas a la población escolar, 1996. Madrid: Delegación del Gobierno para el Plan Nacional sobre Drogas. 1997. 
(7) Plan Nacional sobre Drogas: Encuesta sobre drogas a la población escolar, 1998. PNSD/Internet. 2000.

(8) Plan Nacional sobre Drogas: Encuesta domiciliaria sobre uso de drogas, 1995. Madrid: Delegación del Gobierno para el Plan Nacional sobre Drogas. 1996.

(9) Plan Nacional sobre Drogas: Encuesta domiciliaria sobre uso de drogas, 1997. Madrid: Delegación del Gobierno para el Plan Nacional sobre Drogas. 1998.
(10) Sáiz P.A., González M.P.; Jiménez L.; Delgado Y.; Liboreiro M.J., Granda B., Bobes J.: Consumo de alcohol, tabaco y otras drogas y rasgos de personalidad en jóvenes de enseñanza secundaria. Adicciones 1999; 11 (3): 209-220.

(11) Megías E., Comas D., Elzo J., Navarro J., Romaní O.: La percepción social de los problemas de drogas en España. Madrid: FAD. 2000.

(12) Watson S.J., Benson J.A., Joy J.E.: Marijuana and medicine: assessing the science base. Arch Gen Psychiatry 2000; 57: 547-552. 